Www.jmscr.igmpublication.org

Index Copernicus Value: 79.54

ISSN (e)-2347-176x ISSN (p) 2455-0450

crossref DOI: https://dx.doi.org/10.18535/jmscr/v7i6.96

\title{
Comparison of Low-Dose and Standard-Dose CT in Patients with Clinically Suspected Urolithiasis: A Prospective Study
}

\author{
Authors \\ Manik Mahajan ${ }^{1 *}$, Poonam Sharma ${ }^{2}$ \\ ${ }^{1}$ Lecturer, Department of Radio-Diagnosis \& Imaging, Government Medical College, Jammu \\ ${ }^{2}$ Consultant Pathologist, Atulaya Healthcare, Jammu \\ *Corresponding Author \\ Manik Mahajan \\ House no 109, Sector-7, Channi Himmat, Jammu (J\&K), India - 180015
}

\begin{abstract}
Background: The prevalence and recurrence of urolithiasis are high; particularly in the north-western part of India. Ultrasonography has poor sensitivity and specificity compared to Non Contrast Computed Tomography (CT) for detection of both renal and ureteral calculi. Drawbacks of Non contrast CT include radiation exposure which can be overcome by low-dose CT protocols. Low-dose CT may be a useful investigation with high diagnostic accuracy and less radiation hazards in evaluating urolithiasis.

Aim: To compare efficacy of low-dose non contrast CT with standard-dose CT in the detection of urolithiasis

Material and Methods: Fifty cases of clinically suspected urolithiasis underwent both abdominal standard-dose CT (140 mAs) and low-dose CT (70 mAs) with fixed kVp (120). Patients with BMI <30 (non obese) were included in the study. Both the scans were independently reviewed by two radiologists for the characterization of urolthiasis and any indirect signs of renal and ureteral colic. Results for low-dose CT were compared with those obtained with standard-dose CT (reference standard).

Results: Majority of patients were seen in $3^{\text {rd }}$ decade with male to female ratio of 1:8. Eighty Eight calculi were detected in 50 patients. Low-dose CT had approx. $82 \%$ and $100 \%$ sensitivity in identifying renal calculi $<5 \mathrm{~mm}$ and $>5 \mathrm{~mm}$ size respectively while it had approx. 92\% sensitivity for the detection of ureteric calculi. Mean effective radiation dose in standard-dose CT and low-dose CT was $2.1 \mathrm{mSv}$ and 4.3 $m S v$ respectively.
\end{abstract}

Conclusions: Low-dose CT provides effective method of identifying and evaluating urolthiasis with high diagnostic accuracy and sensitivity and significant dose reduction as compared to standard-dose CT.

\section{Introduction}

Urolithiasis is a common pathology with the lifetime risk exceeding $12 \%$ in men and $6 \%$ in women $^{[1]}$. In addition, recurrence rates are quite high with an estimated rate of $30 \%$ to $40 \%$ within
5 years $20^{[2]}$. The incidence, prevalence and recurrence of urolithiasis are very high; especially in the north-western part of India ${ }^{[3]}$.

Ultrasonography is an attractive investigation in evaluation of urolthiasis because it is less 
expensive, universally available and non-invasive with lack of ionizing radiation ${ }^{[1]}$. However the results are limited due to its image obscuration by underlying bowel loops and bony structures ${ }^{[1]}$. Many studies have demonstrated decreased sensitivity and specificity of Ultrasonography as compared to Computed Tomography (CT) for detection of urolthiasis. CT is now recommended by numerous authors ${ }^{[4,5]}$ as the initial diagnostic imaging technique in patients with suspected renal colic. Non-contrast CT is highly sensitive technique for stone detection. It can be performed rapidly and needs no intravenous iodinated contrast. Many studies have shown a high sensitivity and high specificity of non contrast CT for stone disease and alternative diagnoses are found in $10 \%$ to $24 \%$ of patients with acute flank pain $^{[6]}$.

Many authors have reported that in the screening of patients with suspected renal colic, low-dose CT protocols can be employed which involve substantial reduction of tube current or increased pitch $^{[7-10]}$. Low-dose CT can be performed in short time, does not require any bowel preparation and has lesser cost as compared to conventional $\mathrm{CT}^{[1]}$. It also provides accurate information regarding the stone size, location, density, direct and indirect signs of obstruction and even diagnosis of nonurological conditions ${ }^{[1]}$. Also low-dose CT may achieve sensitivities and specificities close to those reported for standard-dose CT in detecting ureteral stones ${ }^{[11,12]}$. The aim of our study is to compare low-dose CT (70 mAs) with standarddose (140 mAs) non contrast CT for the detection of renal and ureteral calculi.

\section{Material and Methods}

The study was conducted in a tertiary care centre in North West India. Clinically suspected patients of urolithiasis, referred from the urology department for Non contrast CT formed the material of the study. Pregnant females, children and obese patients (Body mass index >30) were excluded. CT was performed with 64 slice-MDCT scanner (Somatom Definition AS+ scanner from
Siemens Healthcare) without oral or intravenous contrast. Scan done from diaphragm to lower symphysis pubis with standard-dose CT (140 mAs) first followed by Low-Dose CT (70 mAs). Fixed tube voltage of $120 \mathrm{kVp}$ was employed in both the scans. Multiplanar 2D and 3D reformatted images were generated on workstations from axial source images. Number, size and site of stones were recorded for both the scans and findings compared. Renal stones were classified according to size ( $<5 \mathrm{~mm}$ and $>5 \mathrm{~mm}$ ) and ureteral stones according to location (upper, middle and lower). Effective dose was calculated for each scan using DLP provided in the report by the manufacturer and multiplying it by constant (k) as prescribed by the European Commission Appendix $\mathrm{C}^{[13]}$ and National Radiological Protection Board $-\mathrm{W} 67^{[14]}$. Mean effective dose was also calculated for both the scans.

\section{Results}

Fifty clinically suspected patients of urolithiasis formed the material of the study. Majority of the patients were seen in $3^{\text {rd }}$ decade of life. Most of the patients were males with male to female ratio of 1:8. Total of 88 calculi were seen on standarddose CT (64 in kidneys and 24 in ureters) (Table 1). Of 64 renal calculi, 22 were $<5 \mathrm{~mm}$ in size while $42>5 \mathrm{~mm}$. Out of 22 renal calculi $(<5 \mathrm{~mm})$ detected on standard-dose CT, 18 were detected on low-dose CT and four were missed likely due to reduced quality of the image. The missed calculi were $<3 \mathrm{~mm}$ in size on standard-dose CT. Also out of 42 renal calculi (> $5 \mathrm{~mm}$ ) detected on standard-dose CT, none was missed on low-dose CT and diagnosed correctly. So sensitivity of lowdose CT in identifying renal calculi $>5 \mathrm{~mm}$ was $100 \%$ while for renal calculi $<5 \mathrm{~mm}$, it was approx. $82 \%$.

24 ureteric calculi were identified in our study on standard-dose CT. Majority of them (66.7\%) were seen in lower ureter. On low-dose CT, 22 ureteric calculi were accurately depicted as compared to standard-dose CT while 2 calculi were missed. The missed calculi were $<3 \mathrm{~mm}$ in diameter and 
located adjacent to iliac crossing. Two pheleboliths were over reported as ureteric calculi on low-dose CT. So sensitivity of low-dose CT in identifying ureteric calculi was approx. $92 \%$.

Effective dose was calculated for all the patients at high and low mAs and mean effective dose calculated. Mean effective dose for standard-dose CT and low-dose CT were $4.3 \mathrm{mSv}$ and $2.1 \mathrm{mSv}$ respectively, implying $>50 \%$ reduction in radiation dose to the patient.

Table 1: Distribution of Calculi $(n=88)$

\begin{tabular}{|l|c|c|}
\hline Calculi & Standard-Dose CT & Low-Dose CT \\
\hline Renal & & \\
A) $<5 \mathrm{~mm}$ & 22 & 18 \\
B) $>5 \mathrm{~mm}$ & 42 & 42 \\
\hline Ureteric & 24 & 22 \\
\hline Total & 88 & 82 \\
\hline
\end{tabular}

\section{Discussion}

Non contrast CT has become the standard imaging technique in patients with renal/ureteric colic. Many reasons are attributed to this including speed, greater sensitivity for stone detection, diagnosis of additional findings and utility in appropriate patient management ${ }^{[15]}$. Only significant disadvantage of $\mathrm{CT}$ is the radiation exposure, particularly in young patients. To decrease radiation dose, various low dose protocols and techniques have been proposed with performance nearly similar to standard-dose $\mathrm{CT}^{[1]}$. This involves significant reduction in the tube current or increased pitch, both of which result in lower radiation dose to patient. Low-dose CT has become the standard for diagnosing urolithiasis with various protocols and low dose settings resulting in a different radiation exposure.

Suspecting the diagnosis of renal colic without having any information about the calculus size and location is usually not sufficient to select the most appropriate therapeutic approach, the need for hospitalization, or the need for extracorporeal shockwave lithotripsy or other urologic procedures $^{[4,16]}$. The size and location of the calculus, particularly ureteric calculi, are determinants of the likelihood of spontaneous stone passage and Characterization of stone morphology and location are the major advantages of CT over other imaging techniques (abdominal radiography, sonography) commonly used in the initial evaluation of patients with suspected renal colic $^{[4,17]}$.

In our study, 88 calculi were detected on standarddose CT, of which 64 were renal calculi and 24 were ureteric calculi. 22 renal calculi were $<5 \mathrm{~mm}$ in size and of these, only 18 were detected on lowdose CT implying a sensitivity of approx. $82 \%$. No renal calculus $>5 \mathrm{~mm}$ was missed on low-dose $\mathrm{CT}$, implying that low-dose CT was equally sensitive to standard-dose $\mathrm{CT}$ for identifying renal calculi $>5 \mathrm{~mm}$ size. Of 24 ureteric calculi seen on standard-dose CT, 22 were detected accurately on low-dose CT while 2 were missed with sensitivity of approx. 92\%. Two phleboliths were overestimated as ureteric calculi on low-dose CT. The missed calculi were $<3 \mathrm{~mm}$ in size and located in lower ureter. It may be stated that calculi $<5 \mathrm{~mm}$ rarely require urologic procedures and $5 \mathrm{~mm}$ is generally the critical size for urology referral as chances of spontaneous passage progressively decreases as a calculus exceeds this size $e^{[18-20]}$. The results of our comparative study, with those obtained from prior series ${ }^{[12,21]}$ suggest that a lowdose CT can be used as the first-line imaging tool in the workup of patients with suspected renal and ureteral colic in non obese patients, providing that clinicians and patients are aware of the limitations and advantages of this technique with regard to standard-dose CT without additional predisposing medical conditions ${ }^{[22]}$.

\section{Conclusions}

Low-dose CT scan is an effective method of identifying and evaluating urinary tract stones. Low-dose CT achieves sensitivities close to those of standard-dose CT for diagnosing renal and ureteral calculi and provides significant dose reduction to patients compared to standard dose CT. Further Low-dose CT may be considered as preferred investigation for the management of urolithiasis, in the scenario of a developing country. 
Conflicts of Interest: Nil

Source of Funding: Nil

Acknowledgements: Nil

\section{References}

1. Sharma S, Chaudhari, Rawal K, Khant S. Low dose computed tomography KUB region for management of urolithiasis in Indian scenario. Int Surg J. 2018 Feb;5(2):638-642.

2. Bhatt K, Monga M, Remer EM. Low-Dose Computed Tomography in the Evaluation of Urolithiasis. J Endourol. 2015 May;29(5):504-11.

3. Ansari MS, Gupta NP, Hemal AK, Dogra PN, Seth A, Aron M, et al. Spectrum of stone composition: Structural analysis of 1050 upper urinary tract calculi from northern India. Int J Urol. 2005 Jan;12(1):12-6.

4. Teichman JM. Clinical practice: acute renal colic from ureteral calculus. N Engl J Med. 2004 Feb 12;350(7):684-93.

5. Abramson $\mathrm{S}$, Walders $\mathrm{N}$, Applegate KE, Gilkeson RC, Robbin MR. Impact in the emergency department of unenhanced CT on diagnostic confidence and therapeutic efficacy in patients with suspected renal colic: a prospective survey. AJR Am J Roentgenol. 2000 Dec;175(6):1689-95.

6. Niemann $T$, Kollmann $T$, Bongartz $G$. Diagnostic performance of low-dose CT for the detection of urolithiasis: A metaanalysis. AJR Am J Roentgenol. 2008 Aug;191(2):396-401.

7. Meagher T, Sukumar VP, Collingwood J, Crawley T, Schofield D, Henson J et al. Low dose computed tomography in suspected acute renal colic. Clin Radiol. 2001 Nov;56(11):873-6.

8. Hamm M, Knopfle E, Wartenberg S, Wawroschek F, Weckermann D, Harzmann R. Low dose unenhanced helical computerized tomography for the evaluation of acute flank pain. J Urol. 2002 Apr;167(4):1687-91.

9. Kim BS, Hwang IK, Choi YW, Namkung S, Kim HC, Hwang WC et al. Low-dose and standard-dose unenhanced helical computed tomography for the assessment of acute renal colic: prospective comparative study. Acta Radiol. 2005 Nov;46(7):756-63.

10. Liu W, Esler SJ, Kenny BJ, Goh RH, Rainbow AJ, Stevenson GW. Low-dose non enhanced helical CT of renal colic: assessment of ureteric stone detection and measurement of effective dose equivalent. Radiology. 2000 Apr;215(1):51-4.

11. Tack D, Sourtzis S, Delpierre I, de Maertelaer V, Gevenois PA. Low-dose unenhanced multidetector CT of patients with suspected renal colic. AJR Am J Roentgenol. 2003 Feb;180(2):305-11.

12. Kluner C, Hein PA, Gralla O, Hein E, Hamm B, Romano V et al. Does ultralowdose $\mathrm{CT}$ with a radiation dose equivalent to that of KUB suffice to detect renal and ureteral calculi? J Comput Assist Tomogr. 2006 Jan-Feb;30(1):44-50.

13. Shrimpton P. Assessment of patient dose in CT. In: EUR. European guidelines for multislice computed tomography funded by the European Commission 2004: contract number FIGMCT2000-20078CT-TIP. Luxembourg, Luxembourg: European Commission, 2004:Appendix C

14. Shrimpton PC, Hillier MC, Lewis MA, Dunn M. Doses from computed tomography (CT) examinations in the UK: 2003 review. Chilton, UK: National Radiological Protection Board, 2005:report NRPB-W67.

15. Şalk İ, Çetin A, Korkmaz İ, Sezer F, Atalar MH. Place of non-contrast computed tomography for evaluation of flank pain and suspected urinary stone in emergency service. Cumhuriyet Med J 2014;36:42-50. 
16. Dalla Palma L, Pozzi-Mucelli R, Stacul F. Presentday imaging of patients with renal colic. Eur Radiol. 2001;11(1):4-17.

17. Dalrymple NC, Verga M, Anderson KR, Bove P, Covey AM, Rosenfield AT et al. The value of unenhanced helical computerized tomography in the management of acute flank pain. J Urol. 1998 Mar;159(3):735-40.

18. Miller OF, Kane CJ. Time to stone passage for observed ureteral calculi: a guide for patient education. J Urol. 1999 Sep;162(3 Pt 1):688-90; discussion 690-1.

19. Varanelli MJ, Coll DM, Levine JA, Rosenfield AT, Smith RC. Relationship between duration of pain and secondary signs of obstruction of the urinary tract on unenhanced helical CT. AJR Am J Roentgenol. 2001 Aug;177(2):325-30.

20. Coll DM, Varanelli MJ, Smith RC. Relationship of spontaneous passage of ureteral calculi to stone size and location as revealed by unenhanced helical CT. AJR Am J Roentgenol. 2002 Jan;178 (1):101-3.

21. Poletti PA, Platon A, Rutschmann OT, Verdun FR, Schmidlin FR, Iselin CE et al. Abdominal plain film in patients admitted with clinical suspicion of renal colic: should it be replaced by low-dose computed tomography? Urology. 2006 Jan;67(1):64-8.

22. Poletti PA, Platon A, Rutschmann OT, Schmidlin FR, Iselin CE, Becker CD . Low-dose versus standard-dose CT protocol in patients with clinically suspected renal colic. AJR Am J Roentgenol. 2007 Apr;188(4):927-33. 